\title{
ON EMBEDDING CONES OVER CIRCULARLY CHAINABLE CONTINUA
}

\author{
RALPH BENNETT AND W. R. R. TRANSUE
}

It is known that every chainable continuum (i.e., every 1-cell-like continuum) can be embedded in the plane [1]. Every 2-cell-like continuum can be embedded in $E^{4}$ [5]. It is natural to ask whether every 2 -cell-like continuum can be embedded in $E^{3}$. Fearnley [3] has stated that the cone over a dyadic solenoid cannot be embedded in $E^{3}$, but no proof has appeared. In this note we show that the cone over a nonplanar circularly chainable continuum cannot be embedded in $E^{3}$. This provides a large class of 2-cell-like continua not embeddable in $E^{3}$.

A definition of what it means to say that a continuum is $P$-like may be found in [6].

For any continuum $M$, the cone over $M$ is the decomposition space $C(M)$ of the upper semicontinuous decomposition of $M \times[0,1]$ whose only nondegenerate element is $M \times\{1\}$. We call the point $M \times\{1\}$ of $C(M)$ the vertex of $C(M)$ and the set of points $\{(m, 0), m$ in $M\}$, we call the base of $C(M)$.

It is easy to see that if $P$ is a continuum and $M$ is $P$-like then $C(M)$ is $C(P)$-like. Therefore every cone over a circularly chainable continuum is 2 -cell-like.

Lemma. Suppose $M$ is a continuum and $S$ is a closed subset of $C(M)$ which separates the vertex of $C(M)$ from the base of $C(M)$. Let $v$ denote the vertex of $C(M), B$ the base of $C(M)$ and $\pi$ the projection of $C(M) \backslash\{v\}$ onto $B$ defined by $\pi(m, t)=(m, 0)$. There is a subcontinuum $D$ of $S$ such that $\pi(D)=B$.

Proof. We may assume that $M$ is embedded in the Hilbert cube $H$ and that $C(M)$ is embedded in $H \times[0,1]$ as $\{(t p, t) \mid t \in[0,1], p \in M\}$. Let $\left\{M_{i}\right\}_{i=1}^{\infty}$ be a sequence of locally connected subcontinua of $H$ such that $M \subset M_{i}$ and $M_{i}$ is contained in the $1 / i$ neighborhood of $M$. Let $S_{i}(i=1,2, \cdots)$ be the closed $1 / i$ neighborhood of $S$ in $H \times[0,1]$. For each $i$ there is an integer $j(i)$ such that $S_{i}$ separates $M_{j(i)}$ from $v$ in $C\left(M_{j(i)}\right)\left(=\left\{(t p, t) \mid t \in[0,1], p \in M_{j(i)}\right\}\right)$. Since $C\left(M_{j(i)}\right)$ is the cone over a locally connected continuum it is locally connected and unicoherent [2, Theorem A, p. 232] and thus some subcontinuum $D_{i}$ of $S_{i}$ separates $M_{j(i)}$ from $v$ in $C\left(M_{j(i)}\right)$ [7, Theorem 4.12, p. 51]. Let

Received by the editors April 29, 1968. 
$D$ be the limit of some convergent subsequence of the sequence $D_{1}, D_{2}, \cdots$. Evidently $D$ is a subcontinuum of $S$. If for some $p$ in $M,\{(t p, t) \mid t \in[0,1]\}$ misses $D$ then this set misses $D_{i}$ for some $i$, contradicting the fact that $D_{i}$ separated $v$ from $M_{j(i)}$ and hence $v$ from $M$.

THEOREM. If $M$ is a nonplanar circularly chainable continuum then $C(M)$ cannot be embedded in $E^{2}$.

Proor. Suppose $h$ is an embedding of $C(M)$ into $E^{z}$. Denote as $B$ the base of $C(M)$ and as $v$ the vertex of $C(M)$. There is a 2-sphere $S$ in $E^{3}$ which separates $h(v)$ from $h(B)$. By the lemma there is a subcontinuum $C$ of the intersection of $S$ and $h(C(M))$ which can be mapped onto $h(B)$. But $C$ could not be all of $S$ since $h(B)$ is not locally connected. W. T. Ingram has shown [4] that there is no map of a planar continuum onto a nonplanar circularly chainable continuum, and we have a contradiction.

\section{REFERENCES}

1. R. H. Bing, Snake-like continua, Duke Math. J. 18 (1951), 643-663.

2. E. Cech, Sur les continus Peaniens unicohtrents, Fund. Math. 20 (1933), 232-243.

3. L. Fearnley, Embedding of topological products of k-cell-like continua, Amer. J. Math. 88 (1966), 347-356.

4. W. T. Ingram, Concerning nomplanar circle-like continua, Canad. J. Math. 19 (1967), 242-250.

5. J. R. Isbell, Embeddings of inverse limits, Ann. of Math. 70 (1959), 73-84.

6. S. Mardesit and J. Segal, e-mappings onto polyhedra, Trans. Amer. Math. Soc. 109 (1963), 146-164.

7. R. L. Wilder, Topology of manifolds, Amer. Math. Soc. Colloq. Publ., Vol. 32, Amer. Math. Soc., Providence, R. I., 1949.

Auburn University 